\title{
Oscillation criteria for third-order neutral dynamic equations with continuously distributed delay
}

Mehmet Tamer Şenel ${ }^{1 *}$ and Nadide Utku²

"Correspondence:

senel@erciyes.edu.tr

1 Department of Mathematics,

Faculty of Sciences, Erciyes

University, Kayseri, 38039, Turkey

Full list of author information is

available at the end of the article

\section{Abstract}

It is the purpose of this paper to give oscillation criteria for the third-order neutral dynamic equations with continuously distributed delay,

$$
\left[r(t)\left(\left[x(t)+\int_{a}^{b} p(t, \eta) x[\tau(t, \eta)] \Delta \eta\right]^{\Delta \Delta}\right)^{\gamma}\right]^{\Delta}+\int_{c}^{d} q(t, \xi) f(x[\phi(t, \xi)]) \Delta \xi=0
$$

on a time scale $\mathbb{T}$, where $\gamma$ is the quotient of odd positive integers. By using a generalized Riccati transformation and an integral averaging technique, we establish some new sufficient conditions which ensure that every solution of this equation oscillates or converges to zero.

Keywords: oscillation; time scales; third-order neutral dynamic equation; asymptotic behavior

\section{Introduction}

We are concerned with the oscillatory behavior of third-order neutral dynamic equations with continuously distributed delay,

$$
\left[r(t)\left(\left[x(t)+\int_{a}^{b} p(t, \eta) x[\tau(t, \eta)] \Delta \eta\right]^{\Delta \Delta}\right)^{\gamma}\right]^{\Delta}+\int_{c}^{d} q(t, \xi) f(x[\phi(t, \xi)]) \Delta \xi=0,
$$

on an arbitrary time scale $\mathbb{T}$, where $\gamma$ is a quotient of odd positive integers. Throughout this paper, we will assume the following hypotheses:

(H1) $r$ and $q$ are positive rd-continuous functions on $\mathbb{T}$ and

$$
\int_{t_{0}}^{\infty}\left(\frac{1}{r(t)}\right)^{\frac{1}{\gamma}} \Delta t=\infty
$$

(H2) $p(t, \eta) \in C_{r d}\left(\left[t_{0}, \infty\right) \times[a, b], \mathbb{R}\right), 0 \leq p(t) \equiv \int_{a}^{b} p(t, \eta) \Delta \eta \leq P<1$;

(H3) $\tau(t, \eta) \in C_{r d}\left(\left[t_{0}, \infty\right) \times[a, b], \mathbb{T}\right)$ is not a decreasing function for $\eta$ and such that

$$
\tau(t, \eta) \leq t \quad \text { and } \quad \lim _{t \rightarrow \infty} \min _{\eta \in[a, b]} \tau(t, \eta)=\infty
$$

O2014 Şenel and Utku; licensee Springer. This is an Open Access article distributed under the terms of the Creative Commons Attribution License (http://creativecommons.org/licenses/by/2.0), which permits unrestricted use, distribution, and reproduction in any medium, provided the original work is properly cited. 
(H4) $\phi(t, \xi) \in C_{r d}\left(\left[t_{0}, \infty\right) \times[c, d], \mathbb{T}\right)$ is not decreasing function for $\xi$ and such that

$$
\phi(t, \xi) \leq t \quad \text { and } \quad \lim _{t \rightarrow \infty} \min _{\xi \in[c, d]} \phi(t, \xi)=\infty
$$

(H5) the function $f \in C_{r d}(\mathbb{T}, \mathbb{R})$ is assumed to satisfy $u f(u)>0$ and there exists a positive rd-continuous function $\delta(t)$ on $\mathbb{T}$ such that $\frac{f(u)}{u^{\gamma}} \geq \delta$, for $u \neq 0$.

Define the function by

$$
z(t)=x(t)+\int_{a}^{b} p(t, \eta) x[\tau(t, \eta)] \Delta \eta
$$

Furthermore, (1) is like the following:

$$
\left[r(t)\left([z(t)]^{\Delta \Delta}\right)^{\gamma}\right]^{\Delta}+\int_{c}^{d} q(t, \xi) f(x[\phi(t, \xi)]) \Delta \xi=0 .
$$

A solution $x(t)$ of (1) is said to be oscillatory if it is neither eventually positive nor eventually negative, otherwise it is non-oscillatory.

Much recent attention has been given to dynamic equations on time scales, or measure chains, and we refer the reader to the landmark paper of Hilger [1] for a comprehensive treatment of the subject. Since then, several authors have expounded various aspects of this new theory; see the survey paper by Agarwal et al. [2]. A book on the subject of time scales by Bohner and Peterson [3] also summarizes and organizes much of the time scale calculus. In the recent years, there has been increasing interest in obtaining sufficient conditions for the oscillation and non-oscillation of solutions of various equations on time scales; we refer the reader to the papers [4-19]. Candan [20] considered oscillation of second-order neutral dynamic equations with distributed deviating arguments of the form

$$
\left(r(t)\left((y(t)+p(t) y(\tau(t)))^{\Delta}\right)^{\gamma}\right)^{\Delta}+\int_{c}^{d} f(t, y(\theta(t, \xi))) \Delta \xi=0
$$

where $\gamma>0$ is a ratio of odd positive integers with $r(t)$ and $p(t)$ real-valued rd-continuous positive functions defined on $\mathbb{T}$. He established some new oscillation criteria and gave sufficient conditions to ensure that all solutions of nonlinear neutral dynamic equation are oscillatory on a time scale $\mathbb{T}$.

To the best of our knowledge, there is very little known about the oscillatory behavior of third-order dynamic equations. Erbe et al. [21] are concerned with the oscillatory behavior of solutions of the third-order linear dynamic equation

$$
x^{\Delta \Delta \Delta}(t)+p(t) x(t)=0
$$

on an arbitrary time scale $\mathbb{T}$, where $p(t)$ is a positive real-valued rd-continuous function defined on $\mathbb{T}$. Li et al. [22] considered third-order nonlinear delay dynamic equation

$$
x^{\Delta^{3}}+p(t) x^{\gamma}(\tau(t))=0 \text {, }
$$

on a time scale $\mathbb{T}$, where $\gamma>0$ is quotient of odd positive integers. 
Erbe et al. [23, 24] established some sufficient conditions which guarantee that every solution of the third-order nonlinear dynamic equation

$$
\left(c(t)\left(a(t) x^{\Delta}(t)\right)^{\Delta}\right)^{\Delta}+q(t) f(x(t))=0,
$$

and the third-order dynamic equation

$$
\left(c(t)\left(\left(a(t) x^{\Delta}(t)\right)^{\Delta}\right)^{\gamma}\right)^{\Delta}+f(t, x(t))=0
$$

oscillate or converge to zero. Li et al. [25] considered the third-order delay dynamic equations

$$
\left(a(t)\left(\left[r(t) x^{\Delta}(t)\right]^{\Delta}\right)^{\gamma}\right)^{\Delta}+f(t, x(\tau(t)))=0,
$$

on a time scale $\mathbb{T}$, where $\gamma>0$ is quotient of odd positive integers, $a$ and $r$ are positive rdcontinuous functions on $\mathbb{T}$, and the so-called delay function $\tau: \mathbb{T} \rightarrow \mathbb{T}$ satisfies $\tau(t) \leq t$, and $\tau(t) \rightarrow \infty$ as $t \rightarrow \infty, f(x) \in C_{r d}(\mathbb{T} \times \mathbb{R}, \mathbb{R})$ is assumed to satisfy $u f(t, u)>0$, for $u \neq 0$, and there exists a function $p$ on $\mathbb{T}$ such that $\frac{f(t, u)}{u^{\gamma}} \geq p(t)>0$, for $u \neq 0$.

Saker [26] considered the third-order nonlinear functional dynamic equations

$$
\left(p(t)\left(\left[r(t) x^{\Delta}(t)\right]^{\Delta}\right)^{\gamma}\right)^{\Delta}+q(t) f(x(\tau(t)))=0,
$$

on a time scale $\mathbb{T}$, where $\gamma>0$ is quotient of odd positive integers. Recently Han et al. [27] and Grace et al. [28] considered the third-order neutral delay dynamic equation

$$
\left(r(t)(x(t)-a(t) x(\tau(t)))^{\Delta \Delta}\right)^{\Delta}+p(t) x^{\gamma}(\delta(t))=0,
$$

on a time scale $\mathbb{T}$.

In this paper, we consider third-order neutral dynamic equation with continuously distributed delay on time scales which is not in literature. We obtain some conclusions which contribute to oscillation theory of third-order neutral dynamic equations.

\section{Several lemmas}

Before stating our main results, we begin with the following lemmas which play an important role in the proof of the main results. Throughout this paper, we let

$$
d_{+}(t):=\max \{0, d(t)\}, \quad d_{-}(t):=\max \{0,-d(t)\},
$$

and

$$
\begin{aligned}
& \beta(t):=b(t), \quad 0<\gamma \leq 1, \quad \beta(t):=b^{\gamma}(t), \quad \gamma>1, \\
& b(t)=\frac{t}{\sigma(t)}, \quad R\left(t, t_{*}\right):=\int_{t_{*}}^{t}\left(\frac{1}{r(s)}\right)^{\frac{1}{\gamma}} \Delta s,
\end{aligned}
$$

where we have sufficiently large $t_{*} \in\left[t_{0}, \infty\right)_{\mathbb{T}}$. 
In order to prove our main results, we will use the formula

$$
\left(z^{\gamma}(t)\right)^{\Delta}=\gamma \int_{0}^{1}\left[h z^{\sigma}+(1-h) z\right]^{\gamma-1} z^{\Delta}(t) d h
$$

where $z(t)$ is delta differentiable and eventually positive or eventually negative, which is a simple consequence of Keller's chain rule (see Bohner and Peterson [3]).

Lemma 2.1 Let $x(t)$ be a positive solution of $(1), z(t)$ is defined as in (3). Then $z(t)$ has only one of the following two properties:

(I) $z(t)>0, z^{\Delta}(t)>0, z^{\Delta \Delta}(t)>0$,

(II) $z(t)>0, z^{\Delta}(t)<0, z^{\Delta \Delta}(t)>0$, with $t \geq t_{1}, t_{1}$ sufficiently large.

Proof Let $x(t)$ be a positive solution of $(1)$ on $\left[t_{0}, \infty\right)$, so that $z(t)>x(t)>0$, and

$$
\left[r(t)\left(z^{\Delta \Delta}(t)\right)^{\gamma}\right]^{\Delta}=-\int_{c}^{d} q(t, \xi) f(x[\phi(t, \xi)]) \Delta \xi<0 .
$$

Then $r(t)\left([z(t)]^{\Delta \Delta}\right)^{\gamma}$ is a decreasing function and therefore eventually of one sign, so $z^{\Delta \Delta}(t)$ is either eventually positive or eventually negative on $t \geq t_{1} \geq t_{0}$. We assert that $z^{\Delta \Delta}(t)>0$ on $t \geq t_{1} \geq t_{0}$. Otherwise, assume that $z^{\Delta \Delta}(t)<0$, then there exists a constant $M>0$, such that

$$
r(t)\left(z^{\Delta \Delta}(t)\right)^{\gamma} \leq-M<0
$$

By integrating the last inequality from $t_{1}$ to $t$, we obtain

$$
z^{\Delta}(t) \leq z^{\Delta}\left(t_{1}\right)-M^{\frac{1}{\gamma}} \int_{t_{1}}^{t}\left(\frac{1}{r(s)}\right)^{\frac{1}{\gamma}} \Delta s .
$$

Let $t \rightarrow \infty$. Then from (H1), we have $(z(t))^{\Delta} \rightarrow-\infty$, and therefore eventually $z^{\Delta}(t)<0$.

Since $z^{\Delta \Delta}(t)<0$ and $z^{\Delta}(t)<0$, we have $z(t)<0$, which contradicts our assumption $z(t)>0$. Therefore, $z(t)$ has only one of the two properties (I) and (II).

This completes the proof.

Lemma 2.2 Let $x(t)$ be an eventually positive solution of (1), correspondingly $z(t)$ has the property (II). Assume that (2) and

$$
\int_{t_{0}}^{\infty} \int_{v}^{\infty}\left[\frac{1}{r(u)} \int_{u}^{\infty} q_{1}(s) \Delta s\right]^{\frac{1}{\gamma}} \Delta u \Delta v=\infty
$$

hold. Then $\lim _{t \rightarrow \infty} x(t)=0$.

Proof Let $x(t)$ be an eventually positive solution of (1). Since $z(t)$ has the property (II), then there exists finite $\lim _{t \rightarrow \infty} z(t)=I$. We assert that $I=0$. Assume that $I>0$, then we have 
$I+\epsilon>z(t)>I$ for all $\epsilon>0$. Choosing $\epsilon<\frac{I(1-P)}{P}$ and using (3) and (H2), we obtain

$$
\begin{aligned}
x(t) & =z(t)-\int_{a}^{b} p(t, \eta)[x(\tau(t, \eta))] \Delta \eta \\
& >I-\int_{a}^{b} p(t, \eta)[x(\tau(t, \eta))] \Delta \eta \\
& \geq I-p(t)[z(\tau(t, a))] \\
& \geq I-P(I+\epsilon)>K z(t),
\end{aligned}
$$

where $K=\frac{I-P(1+\epsilon)}{I+\epsilon}>0$. Using (H5) and (6), we find from (1) that

$$
\begin{aligned}
{\left[r(t)\left(z^{\Delta \Delta}(t)\right)^{\gamma}\right]^{\Delta} } & =-\int_{c}^{d} q(t, \xi) f(x[\phi(t, \xi)]) \Delta \xi \\
& \leq-\int_{c}^{d} q(t, \xi)(x[\phi(t, \xi)])^{\gamma} \delta \Delta \xi \\
& \leq-K^{\gamma} \delta \int_{c}^{d} q(t, \xi)(z[\phi(t, \xi)])^{\gamma} \Delta \xi
\end{aligned}
$$

Note that $z(t)$ has property (II) and (H4), and we have

$$
\left[r(t)\left(z^{\Delta \Delta}(t)\right)^{\gamma}\right]^{\Delta} \leq-K^{\gamma} \cdot \delta \cdot(z[\phi(t, d)])^{\gamma} \int_{c}^{d} q(t, \xi) \Delta \xi=-q_{1}(t)\left(z\left(\phi_{1}(t)\right)\right)^{\gamma}
$$

where $q_{1}(t)=K^{\gamma} \delta \int_{c}^{d} q(t, \xi) \Delta \xi, \phi_{1}(t)=\phi(t, d)$. Integrating inequality (7) from $t$ to $\infty$, we obtain

$$
r(t)\left(z^{\Delta \Delta}(t)\right)^{\gamma} \geq \int_{t}^{\infty} q_{1}(s)\left(z\left(\phi_{1}(s)\right)\right)^{\gamma} \Delta s
$$

Using $\left(z\left(\phi_{1}(s)\right)\right)^{\gamma} \geq I^{\gamma}$, we obtain

$$
z^{\Delta \Delta}(t) \geq \frac{I}{r^{\frac{1}{\gamma}}}\left[\int_{t}^{\infty} q_{1}(s)\right]^{\frac{1}{\gamma}} \Delta(s)
$$

Integrating inequality (8) from $t$ to $\infty$, we have

$$
-z^{\Delta}(t) \geq I \int_{t}^{\infty}\left[\frac{1}{r(u)} \int_{u}^{\infty} q_{1}(s) \Delta(s)\right]^{\frac{1}{\gamma}} \Delta u
$$

Integrating the last inequality from $t_{1}$ to $\infty$, we obtain

$$
z\left(t_{1}\right) \geq I \int_{t_{1}}^{\infty} \int_{v}^{\infty}\left[\frac{1}{r(u)} \int_{u}^{\infty} q_{1}(s) \Delta(s)\right]^{\frac{1}{\gamma}} \Delta u \Delta v
$$

Because (7) and the last inequality contradict (5), we have $I=0$. Since $0 \leq x(t) \leq z(t)$, $\lim _{t \rightarrow \infty} x(t)=0$. This completes the proof. 
Lemma 2.3 Assume that $x(t)$ is a positive solution of $(1), z(t)$ is defined as in (3) such that $z^{\Delta \Delta}(t)>0, z^{\Delta}(t)>0$, on $\left[t_{*}, \infty\right)_{\mathbb{T}}, t_{*} \geq 0$. Then

$$
z^{\Delta}(t) \geq R\left(t, t_{*}\right)^{\frac{1}{\gamma}}(t) z^{\Delta \Delta}(t)
$$

Proof Since $r(t)\left(z^{\Delta \Delta}(t)\right)^{\gamma}$ is strictly decreasing on $\left[t_{*}, \infty\right)_{\mathbb{T}}$, we get for $t \in\left[t_{*}, \infty\right)_{\mathbb{T}}$

$$
\begin{aligned}
z^{\Delta}(t) & >z^{\Delta}(t)-z^{\Delta}\left(t_{*}\right) \\
& =\int_{t_{*}}^{t} \frac{\left(r(s)\left(z^{\Delta \Delta}(t)\right)^{\gamma}\right)^{\frac{1}{\gamma}}}{r^{\frac{1}{\gamma}}(s)} \Delta s \\
& \geq\left(r(t)\left(z^{\Delta \Delta}(t)\right)^{\gamma}\right)^{\frac{1}{\gamma}} \int_{t_{*}}^{t}\left(\frac{1}{r(s)}\right)^{\frac{1}{\gamma}} \Delta s .
\end{aligned}
$$

Using the definition of $R\left(t, t_{*}\right)$, we obtain

$$
z^{\Delta}(t)>R\left(t, t_{*}\right) r^{\frac{1}{\gamma}}(t) z^{\Delta \Delta}(t) \quad \text { on }\left[t_{*}, \infty\right)_{\mathbb{T}} .
$$

Lemma 2.4 Assume that $x(t)$ is a positive solution of (1), correspondingly $z(t)$ has the property (I). Such that $z^{\Delta}(t)>0, z^{\Delta \Delta}(t)>0$, on $\left[t_{*}, \infty\right)_{\mathbb{T}}, t_{*} \geq t_{0}$. Furthermore,

$$
\int_{t_{2}}^{t} q_{2}(s) \phi_{2}^{\gamma}(s) \Delta s=\infty
$$

Then there exists a $T \in\left[t_{*}, \infty\right)_{\mathbb{T}}$, sufficiently large, so that

$$
z(t)>t z^{\Delta}(t)
$$

$z(t) / t$ is strictly decreasing, $t \in[T, \infty)_{\mathbb{T}}$.

Proof Let $U(t)=z(t)-t z^{\Delta}(t)$. Hence $U^{\Delta}(t)=-\sigma(t) z^{\Delta \Delta}(t)<0$. We claim there exists a $t_{1} \in\left[t_{*}, \infty\right)_{\mathbb{T}}$ such that $U(t)>0, z(\phi(t, \xi))>0$ on $\left[t_{1}, \infty\right)_{\mathbb{T}}$. Assume not. Then $U(t)<0$ on $\left[t_{1}, \infty\right)_{\mathbb{T}}$. Therefore,

$$
\left(\frac{z(t)}{t}\right)^{\Delta}=\frac{t z^{\Delta}(t)-z(t)}{t \sigma(t)}=-\frac{U(t)}{t \sigma(t)}>0, \quad t \in\left[t_{1}, \infty\right)_{\mathbb{T}}
$$

which implies that $z(t) / t$ is strictly increasing on $\left[t_{1}, \infty\right)_{\mathbb{T}}$. Pick $t_{2} \in\left[t_{1}, \infty\right)_{\mathbb{T}}$ so that $\phi(t, \xi) \geq \phi\left(t_{1}, \xi\right)$, for $t \geq t_{2}$. Then

$$
\frac{z(\phi(t, \xi))}{\phi(t, \xi)} \geq \frac{z\left(\phi\left(t_{1}, \xi\right)\right)}{\phi\left(t_{1}, \xi\right)}=d>0,
$$

so that $z(\phi(t, \xi))>d \phi(t, \xi)$, for $t \geq t_{2}$. By (1), (3), and (H2), we obtain

$$
\begin{aligned}
x(t) & =z(t)-\int_{a}^{b} p(t, \eta) x[\tau(t, \eta)] \Delta \eta \\
& \geq z(t)-\int_{a}^{b} p(t, \eta) z[\tau(t, \eta)] \Delta \eta
\end{aligned}
$$




$$
\begin{aligned}
& \geq z(t)-z[\tau(t, b)] \int_{a}^{b} p(t, \eta) \Delta \eta \\
& \geq\left(1-\int_{a}^{b} p(t, \eta) \Delta \eta\right) z(t) \\
& \geq(1-P) z(t) .
\end{aligned}
$$

Using (11), (H4), and (H5), we have

$$
\begin{aligned}
{\left[r(t)\left([z(t)]^{\Delta \Delta}\right)^{\gamma}\right]^{\Delta} } & =-\int_{c}^{d} q(t, \xi) f(x[\phi(t, \xi)]) \Delta \xi \\
& \leq-\delta(1-P)^{\gamma} \int_{c}^{d} q(t, \xi) z^{\gamma}(\phi(t, \xi)) \Delta \xi \\
& \leq-\delta(1-P)^{\gamma} z^{\gamma}(\phi(t, c)) \int_{c}^{d} q(t, \xi) \Delta \xi \\
& \leq-q_{2}(t) z^{\gamma}\left(\phi_{2}(t)\right),
\end{aligned}
$$

where $q_{2}(t)=\delta(1-P)^{\gamma} \int_{c}^{d} q(t, \xi) \Delta \xi, \phi_{2}(t)=\phi(t, c)$.

Now by integrating both sides of last equation from $t_{2}$ to $t$, we have

$$
r(t)\left(z^{\Delta \Delta}(t)\right)^{\gamma}-r\left(t_{2}\right)\left(z^{\Delta \Delta}\left(t_{2}\right)\right)^{\gamma}+\int_{t_{2}}^{t} q_{2}(s) z^{\gamma}\left(\phi_{2}(s)\right) \Delta s \leq 0
$$

This implies that

$$
r\left(t_{2}\right)\left(z^{\Delta \Delta}\left(t_{2}\right)\right)^{\gamma} \geq \int_{t_{2}}^{t} q_{2}(s)\left(z\left(\phi_{2}(s)\right)\right)^{\gamma} \Delta s \geq d^{\gamma} \int_{t_{2}}^{t} q_{2}(s) \phi_{2}^{\gamma}(s) \Delta s,
$$

which contradicts (10). So $U(t)>0$ on $t \in\left[t_{1}, \infty\right)_{\mathbb{T}}$ and consequently,

$$
\left(\frac{z(t)}{t}\right)^{\Delta}=\frac{t z^{\Delta}(t)-z(t)}{t \sigma(t)}=-\frac{U(t)}{t \sigma(t)}<0, \quad t \in\left[t_{1}, \infty\right)_{\mathbb{T}}
$$

and we find that $z(t) / t$ is strictly decreasing on $t \in\left[t_{1}, \infty\right)_{\mathbb{T}}$. The proof is now complete.

\section{Main results}

In this section we give some new oscillation criteria for (1).

Theorem 3.1 Assume that (2), (5), and (10) hold. Furthermore, assume that there exists a positive function $\rho \in C_{r d}^{1}\left(\left[t_{0}, \infty\right)_{\mathbb{T}}, \mathbb{R}\right)$, for all sufficiently large $T_{1} \in\left[t_{0}, \infty\right)_{\mathbb{T}}$, there is a $T>T_{1}$ such that

$$
\limsup _{t \rightarrow \infty} \int_{T}^{t}\left[\rho^{\sigma}(s) q_{2}(s)\left(\frac{\phi_{2}(s)}{\sigma(s)}\right)^{\gamma}-\frac{\left(\left(\rho^{\Delta}(s)\right)_{+}\right)^{\gamma+1}}{(\gamma+1)^{\gamma+1}\left(\beta(s) \rho^{\sigma}(s) R\left(s, t_{*}\right)\right)^{\gamma}}\right] \Delta s=\infty \text {. }
$$

Then every solution of $(1)$ is either oscillatory or tends to zero. 
Proof Assume (1) has a non-oscillatory solution $x(t)$ on $\left[t_{0}, \infty\right)_{\mathbb{T}}$. We may assume without loss of generality that $x(t)>0, t \geq t_{1} ; x(\tau(t, \eta))>0,(t, \eta) \in\left[t_{1}, \infty\right) \times[a, b]$ and $x(\phi(t, \xi))>0$, $(t, \xi) \in\left[t_{1}, \infty\right) \times[c, d]$ for all $t_{1} \in\left[t_{0}, \infty\right)_{\mathbb{T}} . z(t)$ is defined as in (3). We suppose that $z(t)>0$. We shall consider only this case, since the proof when $z(t)$ is eventually negative is similar. Therefore Lemma 2.1 and Lemma 2.2, we get

$$
\left[r(t)\left([z(t)]^{\Delta \Delta}\right)^{\gamma}\right]^{\Delta}<0, \quad z^{\Delta \Delta}(t)>0, t \in\left[t_{1}, \infty\right)_{\mathbb{T}},
$$

and either $z^{\Delta}(t)>0$ for $t \geq t_{2} \geq t_{1}$ or $\lim _{t \rightarrow \infty} x(t)=0$. Let $z^{\Delta}(t)>0$ on $\left[t_{2}, \infty\right)_{\mathbb{T}}$.

By (11) and (12), we have

$$
\left[r(t)\left([z(t)]^{\Delta \Delta}\right)^{\gamma}\right]^{\Delta} \leq-q_{2}(t) z^{\gamma}\left(\phi_{2}(t)\right)
$$

where $q_{2}(t)=\delta(1-P)^{\gamma} \int_{c}^{d} q(t, \xi) \Delta \xi, \phi_{2}(t)=\phi(t, c)$.

Define the function $w(t)$ by the Riccati substitution

$$
w(t)=\rho(t) \frac{r(t)\left([z(t)]^{\Delta \Delta}\right)^{\gamma}}{z^{\gamma}(t)} .
$$

Then

$$
\begin{aligned}
w^{\Delta}(t)= & \rho^{\Delta}(t) \frac{r(t)\left([z(t)]^{\Delta \Delta}\right)^{\gamma}}{z^{\gamma}(t)}+\rho^{\sigma}(t)\left[\frac{r(t)\left([z(t)]^{\Delta \Delta}\right)^{\gamma}}{z^{\gamma}(t)}\right]^{\Delta} \\
= & \rho^{\Delta}(t) \frac{r(t)\left([z(t)]^{\Delta \Delta}\right)^{\gamma}}{z^{\gamma}(t)}+\rho^{\sigma}(t) \frac{\left[r(t)\left([z(t)]^{\Delta \Delta}\right)^{\gamma}\right]^{\Delta}}{z^{\gamma \sigma}(t)} \\
& -\rho^{\sigma}(t) \frac{r(t)\left([z(t)]^{\Delta \Delta}\right)^{\gamma}\left(z^{\gamma}(t)\right)^{\Delta}}{z^{\gamma}(t) z^{\gamma \sigma}(t)} .
\end{aligned}
$$

From (1), the definition of $w(t)$ and using the fact $z(t) / t$ is strictly decreasing for $t \in$ $\left[t_{3}, \infty\right)_{\mathbb{T}}, t_{3} \geq t_{2}$, it follows that

$$
\begin{aligned}
& w^{\Delta}(t) \leq \frac{\rho^{\Delta}(t)}{\rho(t)} w(t)-\rho^{\sigma}(t) q_{2}(t) \frac{z^{\gamma}\left(\phi_{2}(t)\right)}{z^{\gamma \sigma}(t)}-\rho^{\sigma}(t) \frac{r(t)\left([z(t)]^{\Delta \Delta}\right)^{\gamma}\left(z^{\gamma}(t)\right)^{\Delta}}{z^{\gamma}(t) z^{\gamma \sigma}(t)}, \\
& w^{\Delta}(t) \leq \frac{\rho^{\Delta}(t)}{\rho(t)} w(t)-\rho^{\sigma}(t) q_{2}(t)\left(\frac{\phi_{2}(t)}{\sigma(t)}\right)^{\gamma}-\rho^{\sigma}(t) \frac{r(t)\left([z(t)]^{\Delta \Delta}\right)^{\gamma}\left(z^{\gamma}(t)\right)^{\Delta}}{z^{\gamma}(t) z^{\gamma \sigma}(t)} .
\end{aligned}
$$

Now we consider the following two cases: $0<\gamma \leq 1$ and $\gamma>1$. In the first case $0<\gamma \leq 1$. Using the Keller chain rule (see [3]), we have

$$
\left(z^{\gamma}(t)\right)^{\Delta}=\gamma \int_{0}^{1}\left[h z^{\sigma}+(1-h) z\right]^{\gamma-1} z^{\Delta}(t) d h \geq \gamma\left(z^{\sigma}(t)\right)^{\gamma-1} z^{\Delta}(t),
$$

in view of (16), Lemma 2.2, Lemma 2.3, and (9), we have

$$
\begin{aligned}
w^{\Delta}(t) & \leq-\rho^{\sigma}(t) q_{2}(t)\left(\frac{\phi_{2}(t)}{\sigma(t)}\right)^{\gamma}+\frac{\left(\rho^{\Delta}(t)\right)_{+}}{\rho(t)} w(t)-\gamma \rho^{\sigma}(t) \frac{r(t)\left(z^{\Delta \Delta}(t)\right)^{\gamma} z^{\Delta}(t) z(t)}{z^{\gamma+1}(t) z^{\sigma}(t)} \\
& \leq-\rho^{\sigma}(t) q_{2}(t)\left(\frac{\phi_{2}(t)}{\sigma(t)}\right)^{\gamma}+\frac{\left(\rho^{\Delta}(t)\right)_{+}}{\rho(t)} w(t)
\end{aligned}
$$




$$
\begin{aligned}
& -\gamma \rho^{\sigma}(t) R\left(t, t_{*}\right) \frac{r^{\frac{\gamma+1}{\gamma}}(t)\left(z^{\Delta \Delta}(t)\right)^{\gamma+1} z(t)}{z^{\gamma+1}(t) z(\sigma(t))} \\
\leq & -\rho^{\sigma}(t) q_{2}(t)\left(\frac{\phi_{2}(t)}{\sigma(t)}\right)^{\gamma}+\frac{\left(\rho^{\Delta}(t)\right)_{+}}{\rho(t)} w(t)-\gamma \rho^{\sigma}(t) R\left(t, t_{*}\right) \frac{t}{\sigma(t)} \frac{w^{\frac{\gamma+1}{\gamma}}(t)}{\rho^{\frac{\gamma+1}{\gamma}}(t)} .
\end{aligned}
$$

In the second case $\gamma>1$. Applying the Keller chain rule, we have

$$
\left(z^{\gamma}(t)\right)^{\Delta}=\gamma \int_{0}^{1}\left[h z^{\sigma}+(1-h) z\right]^{\gamma-1} z^{\Delta}(t) d h \geq \gamma(z(t))^{\gamma-1} z^{\Delta}(t)
$$

in the view of (18), Lemma 2.2, Lemma 2.3, and (9), we have

$$
\begin{aligned}
w^{\Delta}(t) \leq & -\rho^{\sigma}(t) q_{2}(t)\left(\frac{\phi_{2}(t)}{\sigma(t)}\right)^{\gamma}+\frac{\left(\rho^{\Delta}(t)\right)_{+}}{\rho(t)} w(t) \\
& -\gamma \rho^{\sigma}(t) \frac{r(t)\left([z(t)]^{\Delta \Delta}\right)^{\gamma} z^{\Delta}(t) z^{\gamma}(t)}{z^{\gamma+1}(t) z^{\gamma \sigma}(t)}, \\
w^{\Delta}(t) \leq & -\rho^{\sigma}(t) q_{2}(t)\left(\frac{\phi_{2}(t)}{\sigma(t)}\right)^{\gamma}+\frac{\left(\rho^{\Delta}(t)\right)_{+}}{\rho(t)} w(t) \\
& -\gamma \rho^{\sigma}(t)\left(\frac{t}{\sigma(t)}\right)^{\gamma} R\left(t, t_{*}\right) \frac{w^{\frac{\gamma+1}{\gamma}}(t)}{\rho^{\frac{\gamma+1}{\gamma}}(t)} .
\end{aligned}
$$

By (17), (19), and the definition of $b(t)$ and $\beta(t)$, we have, for $\gamma>0$,

$$
w^{\Delta}(t) \leq-\rho^{\sigma}(t) q_{2}(t)\left(\frac{\phi_{2}(t)}{\sigma(t)}\right)^{\gamma}+\frac{\left(\rho^{\Delta}(t)\right)_{+}}{\rho(t)} w(t)-\gamma \rho^{\sigma}(t) \beta(t) R\left(t, t_{*}\right) \frac{w^{\lambda}(t)}{\rho^{\lambda}(t)},
$$

where $\lambda:=\frac{\gamma+1}{\gamma}$. Define $A \geq 0$ and $B \geq 0$ by

$$
\begin{aligned}
& A^{\lambda}:=\gamma \rho^{\sigma}(t) \beta(t) R\left(t, t_{*}\right) \frac{w^{\lambda}(t)}{\rho^{\lambda}(t)}, \\
& B^{\lambda-1}:=\frac{\rho^{\Delta}(t)}{\lambda\left(\gamma \rho^{\sigma}(t) \beta(t) R\left(t, t_{*}\right)\right)^{\frac{1}{\lambda}}} .
\end{aligned}
$$

Then using the inequality [15]

$$
\lambda A B^{\lambda-1}-A^{\lambda} \leq(\lambda-1) B^{\lambda},
$$

which yields

$$
\frac{\left(\rho^{\Delta}(t)\right)_{+}}{\rho(t)} w(t)-\gamma \rho^{\sigma}(t) \beta(t) R\left(t, t_{*}\right) \frac{w^{\lambda}(t)}{\rho^{\lambda}(t)} \leq \frac{\left(\left(\rho^{\Delta}(t)\right)_{+}\right)^{\gamma+1}}{(\gamma+1)^{\gamma+1}\left(\beta(t) \rho^{\sigma}(t) R\left(t, t_{*}\right)\right)^{\gamma}} .
$$

From this last inequality and (20), we find

$$
w^{\Delta}(t) \leq-\rho^{\sigma}(t) q_{2}(t)\left(\frac{\phi_{2}(t)}{\sigma(t)}\right)^{\gamma}+\frac{\left(\left(\rho^{\Delta}(t)\right)_{+}\right)^{\gamma+1}}{(\gamma+1)^{\gamma+1}\left(\beta(t) \rho^{\sigma}(t) R\left(t, t_{*}\right)\right)^{\gamma}} .
$$


Integrating both sides from $T$ to $t$, we get

$$
\int_{T}^{t}\left[\rho^{\sigma}(s) q_{2}(s)\left(\frac{\phi_{2}(s)}{\sigma(s)}\right)^{\gamma}-\frac{\left(\left(\rho^{\Delta}(s)\right)_{+}\right)^{\gamma+1}}{(\gamma+1)^{\gamma+1}\left(\beta(s) \rho^{\sigma}(s) R\left(s, t_{*}\right)\right)^{\gamma}}\right] \Delta s \leq w(T)-w(t) \leq w(T),
$$

which contradicts assumption (13). This completes the proof of Theorem 3.1.

Remark 3.1 From Theorem 3.1, we can obtain different conditions for oscillation of (1) with different choices of $\rho(t)$.

Remark 3.2 The conclusion of Theorem 3.1 remains intact if assumption (13) is replaced by the two conditions

$$
\begin{aligned}
& \limsup _{t \rightarrow \infty} \int_{T}^{t} \rho^{\sigma}(s) q_{2}(s)\left(\frac{\phi_{2}(s)}{\sigma(s)}\right)^{\gamma} \Delta s=\infty, \\
& \limsup _{t \rightarrow \infty} \int_{T}^{t} \frac{\left(\left(\rho^{\Delta}(s)\right)_{+}\right)^{\gamma+1}}{(\gamma+1)^{\gamma+1}\left(\beta(s) \rho^{\sigma}(s) \psi\left(s, t_{*}\right)\right)^{\gamma}} \Delta s<\infty .
\end{aligned}
$$

For example, let $\rho(t)=t$. Now Theorem 3.1 yields the following results.

Corollary 3.1 Assume that (H1)-(H5), (5), and (10) hold.If

$$
\limsup _{t \rightarrow \infty} \int_{T}^{t}\left[\sigma(s) q_{2}(s)\left(\frac{\phi_{2}(s)}{\sigma(s)}\right)^{\gamma}-\frac{1}{(\gamma+1)^{\gamma+1}\left(\beta(s) \sigma(s) R\left(s, t_{*}\right)\right)^{\gamma}}\right] \Delta s=\infty
$$

holds, then every solution (1) is either oscillatory or $\lim _{t \rightarrow \infty} x(t)=0$.

For example, let $\rho(t)=1$. Now Theorem 3.1 yields the following results.

Corollary 3.2 Assume that (H1)-(H5), (5), and (10) hold. If

$$
\limsup _{t \rightarrow \infty} \int_{T}^{t} q_{2}(s)\left(\frac{\phi_{2}(s)}{\sigma(s)}\right)^{\gamma} \Delta s=\infty
$$

then every solution (1) is either oscillatory or $\lim _{t \rightarrow \infty} x(t)=0$.

Theorem 3.2 Assume that (2), (5), and (10) hold. Furthermore, suppose that there exist functions $H, h \in C_{r d}(\mathbb{D}, \mathbb{R})$, where $\mathbb{D} \equiv(t, s): t \geq s \geq t_{0}$ such that

$$
\begin{aligned}
& H(t, t)=0, \quad t \geq 0, \\
& H(t, s)>0, \quad t>s \geq t_{0},
\end{aligned}
$$

and $H$ has a nonpositive continuous $\Delta$-partial derivative $H^{\Delta s}(t, s)$ with respect to the second variable and satisfies

$$
H^{\Delta s}(\sigma(t), s)+H(\sigma(t), \sigma(s)) \frac{\rho^{\Delta}(s)}{\rho(s)}=-\frac{h(t, s)}{\rho(s)} H(\sigma(t), \sigma(s))^{\frac{\gamma}{\gamma+1}},
$$


and for all sufficiently large $T_{1} \in\left[t_{0}, \infty\right)_{\mathbb{T}}$, there is a $T>T_{1}$ such that

$$
\limsup _{t \rightarrow \infty} \frac{1}{H(\sigma(t), T)} \int_{T}^{\sigma(t)} K(t, s)=\infty
$$

where $\rho$ is a positive $\Delta$-differentiable function and

$$
K(t, s)=H(\sigma(t), \sigma(s)) \rho^{\sigma}(s) q_{2}(s)\left(\frac{\phi_{2}(s)}{\sigma(s)}\right)^{\gamma}-\frac{\left(h_{-}(t, s)\right)^{\gamma+1}}{(\gamma+1)^{\gamma+1}\left(\beta(s) \rho^{\sigma}(s) R\left(s, T_{1}\right)\right)^{\gamma}} \Delta s=\infty .
$$

Then every solution of (1) is either oscillatory or tends to zero.

Proof Suppose that $x(t)$ is a non-oscillatory solution of (1) and $z(t)$ is defined as in (3). Without loss of generality, we may assume that there is a $t_{1} \in\left[t_{0}, \infty\right)_{\mathbb{T}}$ sufficiently large so that the conclusions of Lemma 2.1 hold and (24) holds for $t_{2}>t_{1}$. If case (1) of Lemma 2.1 holds then proceeding as in the proof of Theorem 3.1, we see that (20) holds for $t>t_{2}$. Multiplying both sides of (20) by $H(\sigma(t), \sigma(s))$ and integrating from $T$ to $\sigma(t)$, we get

$$
\begin{aligned}
\int_{T}^{\sigma(t)} & H(\sigma(t), \sigma(s)) \rho^{\sigma}(s) q_{2}(s)\left(\frac{\phi_{2}(s)}{\sigma(s)}\right)^{\gamma} \Delta s \\
\leq & -\int_{T}^{\sigma(t)} H(\sigma(t), \sigma(s)) w^{\Delta}(s) \Delta s+\int_{T}^{\sigma(t)} H(\sigma(t), \sigma(s)) \frac{\rho^{\Delta}(s)}{\rho(s)} w(s) \Delta s \\
& -\int_{T}^{\sigma(t)} H(\sigma(t), \sigma(s)) \gamma \rho^{\sigma}(s) \beta(s) R\left(s, T_{1}\right) \frac{w^{\lambda}(s)}{\rho^{\lambda}(s)} \Delta s \quad\left(\lambda=\frac{\gamma+1}{\gamma}\right) .
\end{aligned}
$$

Integrating by parts and using $H(t, t)=0$, we obtain

$$
\int_{T}^{\sigma(t)} H(\sigma(t), \sigma(s)) w^{\Delta}(s) \Delta s=-H(\sigma(t), T) w(T)-\int_{T}^{\sigma(t)} H^{\Delta s}(\sigma(t), s) w(s) \Delta s .
$$

It then follows from (26) that

$$
\begin{aligned}
\int_{T}^{\sigma(t)} & H(\sigma(t), \sigma(s)) \rho^{\sigma}(s) q_{2}(s)\left(\frac{\phi_{2}(s)}{\sigma(s)}\right)^{\gamma} \Delta s \\
\leq & H(\sigma(t), T) w(T)+\int_{T}^{\sigma(t)} H^{\Delta s}(\sigma(t), s) w(s) \Delta s \\
& +\int_{T}^{\sigma(t)} H(\sigma(t), \sigma(s)) \frac{\rho^{\Delta}(s)}{\rho(s)} w(s) \Delta s \\
& -\int_{T}^{\sigma(t)} H(\sigma(t), \sigma(s)) \gamma \rho^{\sigma}(s) \beta(s) R\left(s, T_{1}\right) \frac{w^{\lambda}(s)}{\rho^{\lambda}(s)} \Delta s, \\
\int_{T}^{\sigma(t)} & H(\sigma(t), \sigma(s)) \rho^{\sigma}(s) q_{2}(s)\left(\frac{\phi_{2}(s)}{\sigma(s)}\right)^{\gamma} \Delta s \\
\leq & H(\sigma(t), T) w(T) \\
& +\left[\int_{T}^{\sigma(t)} H H^{\Delta s}(\sigma(t), s)+H(\sigma(t), \sigma(s)) \frac{\rho^{\Delta}(s)}{\rho(s)}\right] w(s) \Delta s \\
& -\int_{T}^{\sigma(t)} H(\sigma(t), \sigma(s)) \gamma \rho^{\sigma}(s) \beta(s) R\left(s, T_{1}\right) \frac{w^{\lambda}(s)}{\rho^{\lambda}(s)} \Delta s .
\end{aligned}
$$


It then follows from (24) that

$$
\begin{aligned}
\int_{T}^{\sigma(t)} & H(\sigma(t), \sigma(s)) \rho^{\sigma}(s) q_{2}(s)\left(\frac{\phi_{2}(s)}{\sigma(s)}\right)^{\gamma} \Delta s \\
\leq & H(\sigma(t), T) w(T) \\
& +\int_{T}^{\sigma(t)}\left[-\frac{h(t, s)}{\rho(s)} H(\sigma(t), \sigma(s))^{\left.\frac{\gamma}{\gamma+1}\right] w(s) \Delta s}\right. \\
& -\int_{T}^{\sigma(t)} H(\sigma(t), \sigma(s)) \gamma \rho^{\sigma}(s) \beta(s) R\left(s, T_{1}\right) \frac{w^{\lambda}(s)}{\rho^{\lambda}(s)} \Delta s \\
\leq & H(\sigma(t), T) w(T)+\int_{T}^{\sigma(t)}\left[\frac{h(t, s)}{\rho(s)} H(\sigma(t), \sigma(s))^{\frac{\gamma}{\gamma+1}}\right] w(s) \Delta s \\
& -\int_{T}^{\sigma(t)} H(\sigma(t), \sigma(s)) \gamma \rho^{\sigma}(s) \beta(s) R\left(s, T_{1}\right) \frac{w^{\lambda}(s)}{\rho^{\lambda}(s)} \Delta s .
\end{aligned}
$$

Therefore, as in Theorem 3.1, by letting

$$
\begin{aligned}
& A^{\lambda}:=H(\sigma(t), \sigma(s)) \gamma \rho^{\sigma}(t) \beta(t) R\left(t, T_{1}\right) \frac{w^{\lambda}(t)}{\rho^{\lambda}(t)}, \\
& B^{\lambda-1}:=\frac{h_{-}(t, s)}{\lambda\left(\gamma \rho^{\sigma}(t) \beta(t) R\left(t, T_{1}\right)\right)^{\frac{1}{\lambda}}} .
\end{aligned}
$$

Then using the inequality [15]

$$
\lambda A B^{\lambda-1}-A^{\lambda} \leq(\lambda-1) B^{\lambda} .
$$

We have

$$
\begin{aligned}
& \int_{T}^{\sigma(t)}\left[\frac{h_{-}(t, s)}{\rho(s)} H(\sigma(t), \sigma(s))^{\frac{\gamma}{\gamma+1}}\right] w(s) \Delta s \\
& \quad-\int_{T}^{\sigma(t)} H(\sigma(t), \sigma(s)) \gamma \rho^{\sigma}(s) \beta(s) R\left(s, T_{1}\right) \frac{w^{\lambda}(s)}{\rho^{\lambda}(s)} \Delta s \\
& \quad=\int_{T}^{\sigma(t)} \frac{\left(h_{-}(t, s)\right)^{\gamma+1}}{(\gamma+1)^{\gamma+1}\left(\beta(s) \rho^{\sigma}(s) R\left(t, T_{1}\right)\right)^{\gamma}} \Delta s, \\
& \int_{T}^{\sigma(t)} H(\sigma(t), \sigma(s)) \rho^{\sigma}(s) q_{2}(s)\left(\frac{\phi_{2}(s)}{\sigma(s)}\right)^{\gamma} \Delta s \\
& \quad \leq H(\sigma(t), T) w(T)+\int_{T}^{\sigma(t)} \frac{\left(h_{-}(t, s)\right)^{\gamma+1}}{(\gamma+1)^{\gamma+1}\left(\beta(s) \rho^{\sigma}(s) R\left(t, T_{1}\right)\right)^{\gamma}} \Delta s .
\end{aligned}
$$

Then for $T>T_{1}$ we have

$$
\begin{aligned}
& \int_{T}^{\sigma(t)}\left[H(\sigma(t), \sigma(s)) \rho^{\sigma}(s) q_{2}(s)\left(\frac{\phi_{2}(s)}{\sigma(s)}\right)^{\gamma}-\frac{\left(h_{-}(t, s)\right)^{\gamma+1}}{(\gamma+1)^{\gamma+1}\left(\beta(s) \rho^{\sigma}(s) R\left(s, T_{1}\right)\right)^{\gamma}}\right] \Delta s \\
& \quad \leq H(\sigma(t), T) w(T),
\end{aligned}
$$


and this implies that

$$
\begin{gathered}
\frac{1}{H(\sigma(t), T)} \int_{T}^{\sigma(t)}\left[H(\sigma(t), \sigma(s)) \rho^{\sigma}(s) q_{2}(s)\left(\frac{\phi_{2}(s)}{\sigma(s)}\right)^{\gamma}\right. \\
\left.-\frac{\left(h_{-}(t, s)\right)^{\gamma+1}}{(\gamma+1)^{\gamma+1}\left(\beta(s) \rho^{\sigma}(s) R\left(s, T_{1}\right)\right)^{\gamma}}\right] \Delta s<w(T),
\end{gathered}
$$

for all large $T$, which contradicts (25). This completes the proof of Theorem 3.2.

Remark 3.3 The conclusion of Theorem 3.2 remains intact if assumption (25) is replaced by the two conditions

$$
\begin{aligned}
& \limsup _{t \rightarrow \infty} \frac{1}{H(\sigma(t), T)} \int_{T}^{\sigma(t)} H(\sigma(t), \sigma(s)) \rho^{\sigma}(s) q_{2}(s)\left(\frac{\phi_{2}(s)}{\sigma(s)}\right)^{\gamma} \Delta s=\infty, \\
& \liminf _{t \rightarrow \infty} \frac{1}{H(\sigma(t), T)} \int_{T}^{\sigma(t)} \frac{\left(h_{-}(t, s)\right)^{\gamma+1}}{(\gamma+1)^{\gamma+1}\left(\beta(s) \rho^{\sigma}(s) R\left(s, T_{1}\right)\right)^{\gamma}} \Delta s<\infty .
\end{aligned}
$$

Remark 3.4 Define $w$ as (14), we also get

$$
w^{\Delta}(t)=r^{\sigma}(t)\left(z^{\Delta \Delta}(t)\right)^{\gamma \sigma}\left[\frac{\rho(t)}{z^{\gamma}(t)}\right]^{\Delta}+\frac{\rho(t)}{z^{\gamma}}\left[r(t)\left(z^{\Delta \Delta}(t)\right)^{\gamma}\right]^{\Delta},
$$

similar to the proofs of Theorem 3.1, we can obtain different results. We leave the details to the reader.

Example 3.1 Consider the following third-order neutral dynamic equation $t \in\left[t_{0}, \infty\right)_{\mathbb{T}}$ :

$$
\left(x(t)+\int_{a}^{b} e^{-t} x(t-\eta) \Delta \eta\right)^{\Delta \Delta \Delta}+\int_{c}^{d} \frac{\beta \cdot t}{\left(t^{2}-t \xi\right)\left(t^{2}-t \xi\right)^{\sigma}} x(t-\xi) \Delta \xi=0,
$$

where $\gamma=1, r(t)=1, \tau(t, \eta)=t-\eta, \phi(t, \xi)=t-\xi, \delta=1, q_{2}(t)=\frac{\beta}{t \phi_{2}(t)}, p(t, \eta)=e^{-t}, q(t, \xi)=$ $\beta \cdot t /\left(t^{2}-t \xi\right)\left(t^{2}-t \xi\right)^{\sigma}$.

It is clear that condition (2), (5), and (10) hold. Therefore, by Theorem 3.1, picking $\rho(t)=t$, we have

$$
\begin{aligned}
& \limsup _{t \rightarrow \infty} \int_{T}^{t}\left[\rho^{\sigma}(s) q_{2}(s)\left(\frac{\phi_{2}(s)}{\sigma(s)}\right)^{\gamma}-\frac{\left(\left(\rho^{\Delta}(s)\right)_{+}\right)^{\gamma+1}}{(\gamma+1)^{\gamma+1}\left(\beta(s) \rho^{\sigma}(s) R\left(s, t_{*}\right)\right)^{\gamma}}\right] \Delta s \\
& \quad=\limsup _{t \rightarrow \infty} \int_{T}^{t}\left[\frac{\beta}{s}-\frac{1}{(\gamma+1)^{(\gamma+1)} s\left(s-t_{*}\right)}\right] \Delta s=\infty .
\end{aligned}
$$

Hence, by Theorem 3.1 every solution of (27) is oscillatory or tends to zero if $\beta>0$.

Example 3.2 Consider the following third-order neutral dynamic equation $t \in\left[t_{0}, \infty\right)_{\mathbb{T}}$ :

$$
\left[\frac{1}{t}\left(\left[x(t)+\int_{a}^{b} \frac{1}{2} x\left[\tau\left(\frac{t}{2}\right)\right] \Delta \eta\right]^{\Delta \Delta}\right)^{3}\right]^{\Delta}+\int_{c}^{d} q(t, \xi) f\left(x\left[\phi\left(\frac{t}{2}\right)\right]\right) \Delta \xi=0
$$

where $\gamma=3, r(t)=\frac{1}{t}, \tau(t, \eta)=\frac{t}{2}, \phi(t, \xi)=\frac{t}{2}, \delta=1, q_{2}(t)=\frac{\beta}{t} \frac{\sigma^{3}(s)}{\phi_{2}^{3}(t)}, p(t, \eta)=\frac{1}{2}$. 
It is clear that condition (2), (5), and (10) hold. Therefore, by Theorem 3.1, picking $\rho(t)=1$, we have

$$
\underset{t \rightarrow \infty}{\limsup } \int_{T}^{t} q_{2}(s)\left(\frac{\phi_{2}(s)}{\sigma(s)}\right)^{3} \Delta s=\limsup _{t \rightarrow \infty} \int_{T}^{t} \frac{\beta}{s} \Delta s=\infty
$$

Hence, by Theorem 3.1 every solution of (28) is oscillatory or tends to zero if $\beta>0$.

\section{Competing interests}

The authors declare that they have no competing interests.

\section{Authors' contributions}

All authors contributed equally to the writing of this paper. All authors read and approved the final manuscript.

\section{Author details}

${ }^{1}$ Department of Mathematics, Faculty of Sciences, Erciyes University, Kayseri, 38039, Turkey. ${ }^{2}$ Institute of Sciences, Erciyes University, Kayseri, 38039, Turkey.

Received: 17 January 2014 Accepted: 19 July 2014 Published: 05 Aug 2014

\section{References}

1. Hilger, S: Analysis on measure chains a unified approach to continuous and discrete calculus. Results Math. 18, 18-56 (1990)

2. Agarwal, RP, Bohner, M, O'Regan, D, Peterson, A: Dynamic equations on time scales: a survey. J. Comput. Appl. Math $141,1-26(2002)$

3. Bohner, M, Peterson, A: Dynamic Equations on Time Scales: An Introduction with Applications. Birkhäuser, Boston (2001)

4. Agarwal, RP, O' Regan, D, Saker, SH: Oscillation criteria for second-order nonlinear neutral delay dynamic equations. J. Math. Anal. Appl. 300(1), 203-217 (2004)

5. Şahiner, Y: Oscillation of second-order neutral delay and mixed-type dynamic equations on time scales. Adv. Differ. Equ. 2006, Article ID 65626 (2006)

6. Wu, H-W, Zhuang, R-K, Mathsen, RM: Oscillation criteria for second-order nonlinear neutral variable delay dynamic equations. Appl. Math. Comput. 178(2), 321-331 (2006)

7. Saker, SH: Oscillation of second-order nonlinear neutral delay dynamic equations on time scales. J. Comput. Appl. Math. 187(2), 123-141 (2006)

8. Zhang, SY, Wang, QR: Oscillation of second-order nonlinear neutral dynamic equations on time scales. Appl. Math. Comput. 216(10), 2837-2848 (2010)

9. Şenel, MT: Kamenev-type oscillation criteria for the second-order nonlinear dynamic equations with damping on time scales. Abstr. Appl. Anal. 2012, Article ID 253107 (2012)

10. Şenel, MT: Oscillation theorems for dynamic equation on time scales. Bull. Math. Anal. Appl. 3, 101-105 (2011)

11. Li, T, Agarwal, RP, Bohner, M: Some oscillation results for second-order neutral dynamic equations. Hacet. J. Math. Stat. 41, 715-721 (2012)

12. Erbe, L, Hassan, TS, Peterson, A: Oscillation of third order nonlinear functional dynamic equations on time scales. Differ. Equ. Dyn. Syst. 18, 199-227 (2010)

13. Şenel, MT: Behavior of solutions of a third-order dynamic equation on time scales. J. Inequal. Appl. 2013, Article ID 47 (2013)

14. Zhang, Q, Gao, L, Yu, Y: Oscillation criteria for third order neutral differential equations with continuously distributed delay. Appl. Math. Lett. 10, 10-16 (2012)

15. Hardy, GH, Littlewood, JE, Pólya, G: Inequalities. Reprint of the 1952 edition. Cambridge Mathematical Library. Cambridge University Press, Cambridge (1988)

16. Graef, J, Saker, SH: Oscillation of third-order nonlinear neutral functional dynamic equations. Dyn. Syst. Appl. 21, 583-606 (2012)

17. Saker, SH: On oscillation of a certain class of third-order nonlinear functional dynamic equations on time scales. Bull. Math. Soc. Sci. Math. Roum. 54, 365-389 (2011)

18. Saker, SH: Oscillation Theory of Dynamic Equations on Time Scales. Lambert Academic Publishing, Colne (2010)

19. Agarwal, RP, O'Regan, D, Saker, SH: Philos-type oscillation criteria of second-order half-linear dynamic equations on time scales. Rocky Mt. J. Math. 37, 1085-1104 (2007)

20. Candan, T: Oscillation of second order nonlinear neutral dynamic equations on time scales with distributed deviating arguments. Comput. Math. Appl. 62, 4118-4125 (2011)

21. Erbe, L, Peterson, A, Saker, SH: Hille and Nehari type criteria for third-order dynamic equations. J. Math. Anal. Appl. $329,112-131(2007)$

22. Li, T, Han, Z, Zhang, C, Sun, Y: Oscillation criteria for third-order nonlinear delay dynamic equations on time scales. Bull. Math. Anal. Appl. 3, 52-60 (2011)

23. Erbe, L, Peterson, A, Saker, SH: Asymptotic behavior of solutions of a third-order nonlinear dynamic equation on time scales. J. Comput. Appl. Math. 181, 92-102 (2005)

24. Erbe, L, Peterson, A, Saker, SH: Oscillation and asymptotic behavior of a third-order nonlinear dynamic equation. Can. Appl. Math. Q. 14(2), 124-147 (2006) 
25. Li, T, Han, Z, Sun, S, Zhao, Y: Oscillation results for third order nonlinear delay dynamic equations on time scales. Bull. Malays. Math. Soc. 34, 639-648 (2011)

26. Saker, SH: Oscillation of third-order functional dynamic equations on time scales. Sci. China Math. 54, 2597-2614 (2011)

27. Han, Z, Li, T, Sun, S, Zhang, C: Oscillation behavior of third order neutral Emden-Fowler delay dynamic equations on time scales. Adv. Differ. Equ. 2010, Article ID 586312 (2010)

28. Grace, SR, Graef, JR, El-Beltagy, MA: On the oscillation of third order delay dynamic equations on time scales. Appl. Math. Comput. 63, 775-782 (2012)

10.1186/1687-1847-2014-220

Cite this article as: Şenel and Utku: Oscillation criteria for third-order neutral dynamic equations with continuously distributed delay. Advances in Difference Equations 2014, 2014:220 